Article

\title{
Transient Wave Scattering and Its Influence on Transient Analysis and Leak Detection in Urban Water Supply Systems: Theoretical Analysis and Numerical Validation
}

\author{
Huan-Feng Duan \\ Department of Civil and Environmental Engineering, The Hong Kong Polytechnic University, Hung Hom, \\ Kowloon, Hong Kong 999077, China; hf.duan@polyu.edu.hk; Tel.: +852-3400-8449
}

Received: 8 September 2017; Accepted: 11 October 2017; Published: 13 October 2017

\begin{abstract}
This paper investigates the impacts of non-uniformities of pipe diameter (i.e., an inhomogeneous cross-sectional area along pipelines) on transient wave behavior and propagation in water supply pipelines. The multi-scale wave perturbation method is firstly used to derive analytical solutions for the amplitude evolution of transient pressure wave propagation in pipelines, considering regular and random variations of cross-sectional area, respectively. The analytical analysis is based on the one-dimensional (1D) transient wave equation for pipe flow. Both derived results show that transient waves can be attenuated and scattered significantly along the longitudinal direction of the pipeline due to the regular and random non-uniformities of pipe diameter. The obtained analytical results are then validated by extensive 1D numerical simulations under different incident wave and non-uniform pipe conditions. The comparative results indicate that the derived analytical solutions are applicable and useful to describe the wave scattering effect in complex pipeline systems. Finally, the practical implications and influence of wave scattering effects on transient flow analysis and transient-based leak detection in urban water supply systems are discussed in the paper.
\end{abstract}

Keywords: water supply pipeline; transient wave; non-uniformities; wave scattering; transient modelling; leak detection

\section{Introduction}

Wave scattering has been commonly studied in shallow water fields where the water waves propagate through the channel bottom with randomly varying bars, as depicted in Figure 1 [1]. Experimental results for such cases together with some theoretical considerations are investigated and discussed in [2,3]. The results in these studies showed that, with the existence of random non-uniformities (inhomogeneities) of channel bottom elevation, eventually the amplitude of the generated wave decreases along the longitudinal direction and it tends to zero if the longitudinal distance is large enough.

Similar random non-uniformities can be found in pipelines (closed conduits) such as random variations in the pipe cross-sectional area (pipe diameter), with respect to length, developing with age (refer to Figure 2). In practical systems, many factors can attribute to the random non-uniformities of pipe cross-sectional area, for example, bio-film build up, corrosion, and deposition in water supply pipes, drainage pipes, crude oil pipes, and arterial systems. In particular, in water pipelines, non-uniformities of pipe diameter may be induced by various different factors as shown in Figure 3 , including corrosion, sediment, junctions and complex connections. 


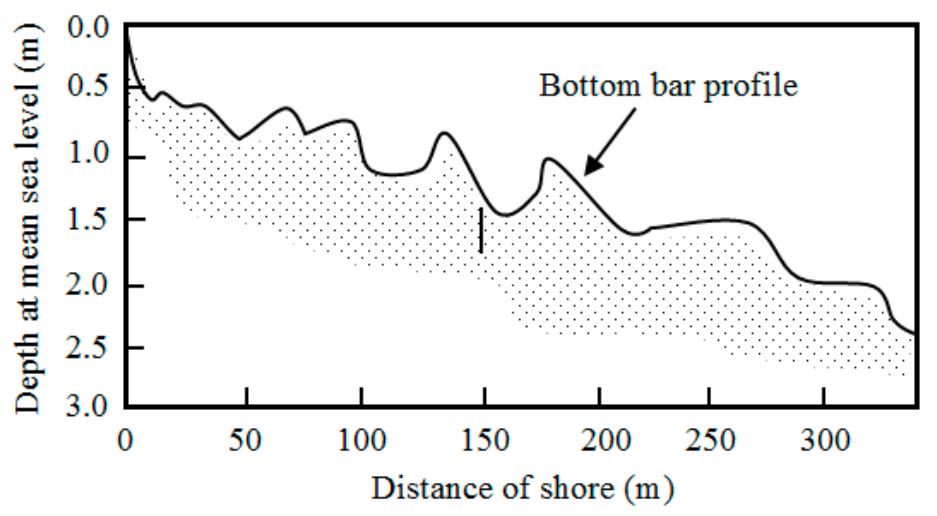

Figure 1. Bottom bar profile in Chesapeake Bay (adapted from [1]).
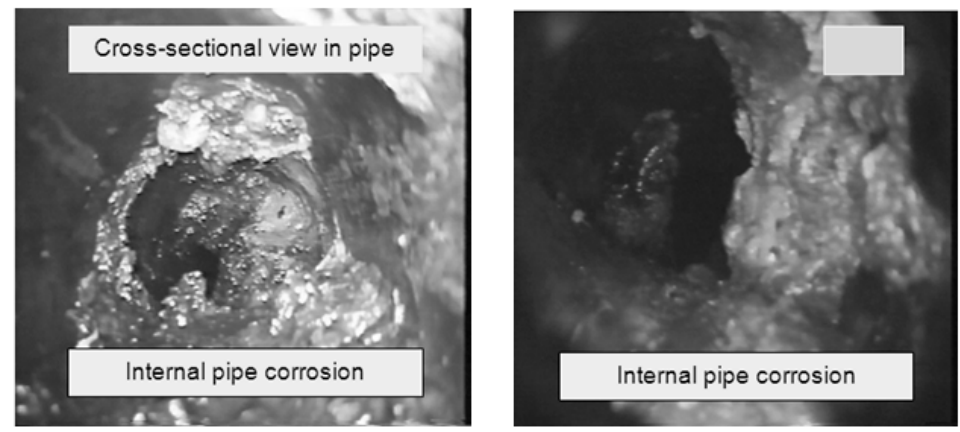

Figure 2. Cross-sectional views of aged water pipelines (adapted from [4]).
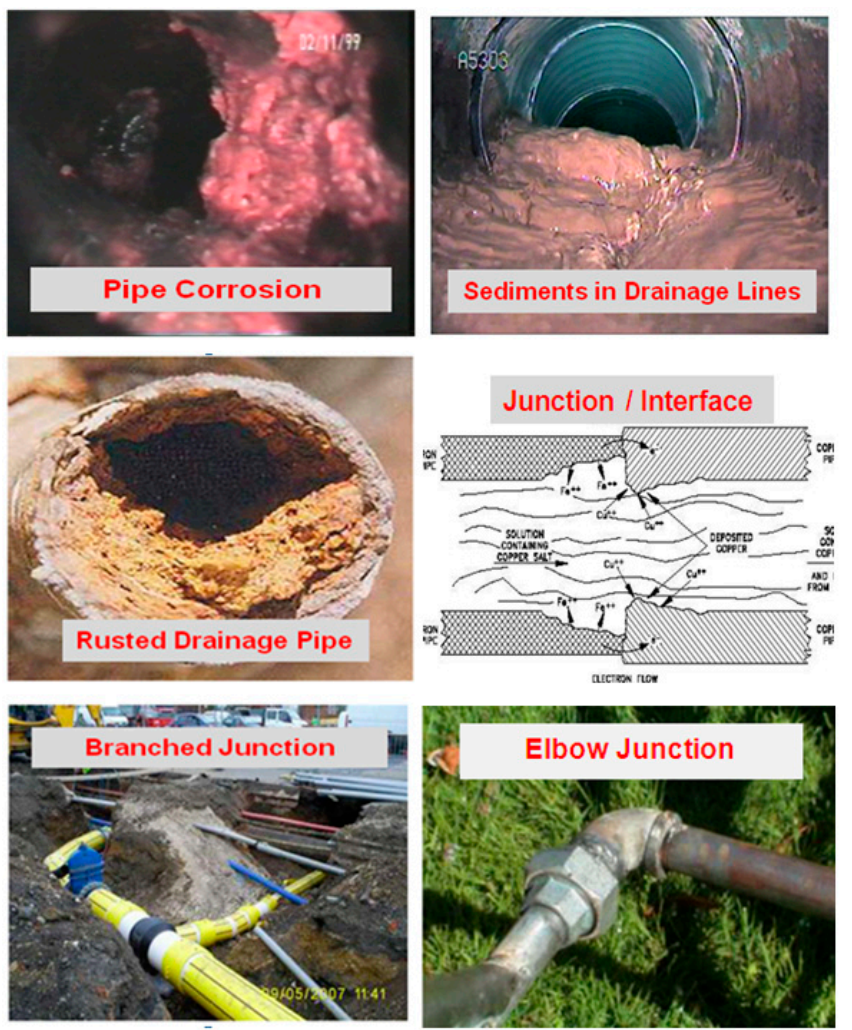

Figure 3. Different factors attributed to non-uniformities of pipe diameter (pictures adapted from online public sources on google websites). 
From the perspective of steady flow in water piping systems, pipe diameter non-uniformities may cause additional energy losses and, thus, require more pumping capacity. On the other hand, from the perspective of unsteady flow, random variations in pipe diameters can result in random reflections and damping of waterhammer waves. So far, the phenomenon of wave scattering in water piping systems and its implications for the structural integrity and robustness of such systems are still not well understood. Recent numerical and experimental studies [4,5] have demonstrated the significant influence of irregular pipe diameters (e.g., roughness and blockage) on the transient wave propagation behaviors, and also on the transient-based defect detection methods (e.g., leakage and blockage). Moreover, their results also indicate that wave scattering by pipe diameter non-uniformities is more important and influential than the corresponding friction effect induced by roughness/irregularities to the wave damping and reflection in both the time and frequency domains. Therefore, an in-depth understanding of such wave scattering effects due to pipe diameter non-uniformities is necessary and critical to transient modelling, analysis and application in water supply pipe systems.

In fact, the wave scattering phenomenon and its relevant influence has been widely studied in many different application fields in the literature such as condensed matter, electromagnetism, seismology and fluid mechanics. For example, a classic paper [6] showed that random impurities have an important consequence on the propagation of electrons in a dirty crystal: the diffusive motion of electrons is terminated and all electrons become localized (this phenomenon is known as Anderson localization). Anderson's idea was used later in the analysis of the propagation of surface waves over a random seabed. Experimental results from previous studies [1,2] demonstrated the localization of water waves over the rough bottom. The analogy between water wave dynamics and Anderson localization is pointed out in [3]. Furthermore, the behavior and propagation of slowly modulated waves in random media has been studied in [7]. However, so far there is no such systematic analysis and theoretical investigation of the transient wave scattering phenomenon in urban water supply pipelines, although it is widely observed from laboratory experiments and field tests in this research area $[4,5,8]$.

To investigate the potential wave-scattering phenomenon induced by random pipe diameter non-uniformities (i.e., pipe cross-sectional area) and to understand its impact on the transient wave propagation in water piping systems, in the present paper the method of multi-scale perturbation from the literature is firstly applied to one-dimensional (1D) waterhammer equations, which describe the flow dynamics in a pipe under the additional assumption that the pipe cross-sectional area (diameter) varies in a random manner along the longitudinal coordinate. Two cases-regular and random pipe non-uniformities-are considered for the analytical derivations. The obtained analytical results are then compared and validated by numerical simulations, which are achieved by the step-discretization approximation for different pipe non-uniformities. Thereafter, further discussion of the practical implications of the results and findings in this study to transient system modelling and pipe leak detection is performed in the paper. Finally, relevant conclusions are drawn at the end of this study.

\section{Problem Statement and Study Framework}

In realistic water supply pipelines, the non-uniformities of pipe diameters could be formed by various different reasons, as shown in Figure 3, resulting in relatively random geometries and distributions of such non-uniformities, as sketched in Figure 4a. In many theoretical studies (e.g., [4,5]), these random non-uniformities are usually treated approximately as different regular shapes or their combinations, in order to conduct mathematical operations and numerical computations. For example, Figure $4 \mathrm{~b}$ with a relatively smooth variation (e.g., sinusoidal shape) and Figure $4 \mathrm{c}$ with relatively sharp variation (e.g., step shape) are two commonly used approximations. From the perspective of mathematics, the complicated random situation in Figure 4a could be a superposition of different numbers of simplified cases in Figure $4 \mathrm{~b}$,c. Therefore, it is a good start to investigate and understand the simplified cases, which can provide insights and a basis to explore and explain more complicated situations, such as the random case in Figure 4a. 


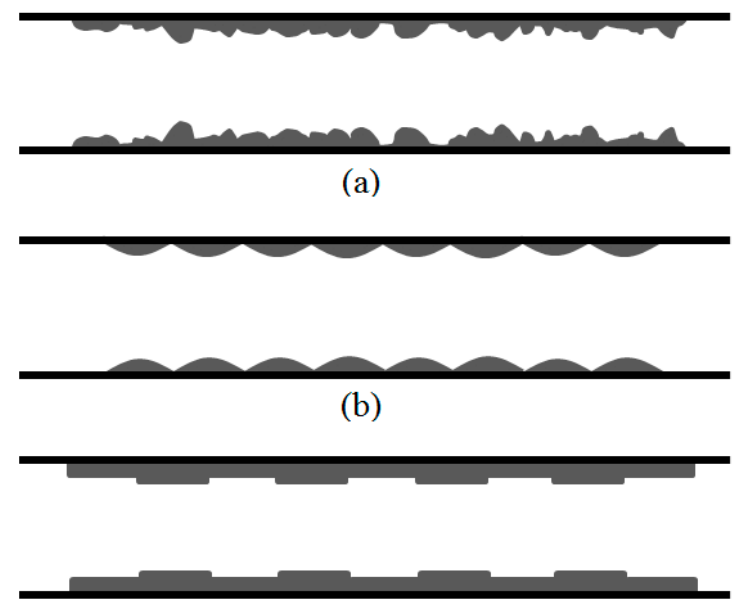

(c)

Figure 4. Sketch of different types of pipe diameter non-uniformities (side-sectional profile): (a) realistic and random situation; (b) regular approximation by sinusoidal variation; (c) regular approximation by step variation.

To this end, in this study, the regular case of pipe diameter non-uniformities in Figure $4 \mathrm{~b}$ is used for preliminary analytical analysis for transient wave propagation in pipelines, while the random case in Figure 4a is applied for further analytical derivations so as to obtain the complete characteristics of transient flows in realistic pipelines. Thereafter, the other regular case in Figure $4 \mathrm{c}$ is adopted as a discrete approximation of a random case in order to achieve numerical simulations for the validation of the derived analytical results in this study. The detailed settings for such numerical simulations are provided later in the part covering numerical applications. The obtained analytical and numerical results are finally discussed for an in-depth understanding of the transient wave behavior and propagation in non-uniform pipelines in urban water supply systems.

\section{Models and Methods}

For clarity, the main models and analysis methods used in this study for investigating transient wave scattering effect in water supply pipelines are summarized as follows.

\subsection{One-Dimentional (1D) Transient Model}

The continuity and momentum equations of the 1D waterhammer model for compressible pipe flow with pipe diameter non-uniformities (i.e., varying pipe cross-sectional area) by neglecting the friction and visco-elastic effects are considered herein [4,9],

$$
\begin{gathered}
\frac{\partial(\rho A)}{\partial t}+\frac{\partial(\rho Q)}{\partial x}=0, \\
\frac{\partial(\rho Q)}{\partial t}+A \frac{\partial P}{\partial x}+\tau_{w} \pi D=0,
\end{gathered}
$$

where $\rho$ is fluid density; $A=A(x)$ is pipe cross-sectional area; $D=D(x)$ is pipe diameter; $Q=Q(x, t)$ is pipe discharge; $P=P(x, t)$ is pressure; $\tau_{w}$ is wall shear stress; $x$ is spatial coordinate; and $t$ is temporal coordinate. In the numerical simulations, the wall shear stress is modelled by the Darcy-Weisbach formula, where only the steady state friction is included. The method of characteristics (MOC) is used for the 1D numerical simulations in this study, and the details for implementing this numerical scheme into above transient model can refer to the classic textbooks and references in this field $[9,10]$. While in the analytical analysis, the friction effect (wall shear stress term in the equation) is excluded due to the 
mathematical complexity and difficulty of analytical derivation, and so as to highlight the effect of wave scattering during transient flow process.

For analytical derivation, the continuity and momentum Equations (1) and (2) can be further lumped into wave equation form through following transformation,

$$
\begin{gathered}
\frac{\partial^{2} P}{\partial t^{2}}=-\frac{\rho a^{2}}{A} \frac{\partial^{2} Q}{\partial x \partial t^{\prime}} \\
\rho \frac{\partial^{2} Q}{\partial x \partial t}=-\frac{\partial}{\partial x}\left(A \frac{\partial P}{\partial x}\right) .
\end{gathered}
$$

where $a$ is acoustic wave speed. After the mathematical elimination operation, the result becomes,

$$
A \frac{\partial^{2} P}{\partial t^{2}}=a^{2} \frac{\partial}{\partial x}\left(A \frac{\partial P}{\partial x}\right)
$$

with the pipe cross-sectional area $A(x)$ varying with $x$. Furthermore, Equation (5) can also be rewritten as,

$$
\underbrace{\frac{\partial^{2} P}{\partial t^{2}}=a^{2} \frac{\partial}{\partial x}\left(A \frac{\partial P}{\partial x}\right)}_{(a)}+\underbrace{(1-A) \frac{\partial^{2} P}{\partial t^{2}}}_{(b)},
$$

where part (a) in Equation (6) has a similar form solved in previous studies for shallow surface wave problems [7], while the other part (b) of Equation (6) is an additional term originated from the case of pressurized wave propagation in water supply pipelines that is focused and dealt with in this study. Similarly, the multi-scale perturbation method from previous studies is further adapted and applied to solve this transient wave equation for pressurized water pipelines $[7,11]$, which is elaborated in the following section.

\subsection{Multi-Scale Perturbation Method}

The method of multi-scale perturbation used in this study follows the previous studies [7,11], with three sets of coordinate scales in both spatial and time introduced as follows:

$$
\begin{gathered}
\text { spatial domain: } x, x_{1}=\varepsilon x, x_{2}=\varepsilon^{2} x, \\
\text { time domain: } t, t_{1}=\varepsilon t, t_{2}=\varepsilon^{2} t,
\end{gathered}
$$

where the three scales $\left(x, x_{1}, x_{2}\right.$ and $\left.t, t_{1}, t_{2}\right)$ correspond to wave oscillations, initial wave modulation, and the modulation by randomness when waves propagate along the pipeline, respectively; $\varepsilon$ characterizes the ratio of different scales and $\varepsilon<<1$. The derivatives with respect to $x$ and $t$ are transformed in accordance with the chain rule as [11],

$$
\begin{gathered}
x=x ; x_{1}=\varepsilon x ; x_{2}=\varepsilon^{2} x ; t=t ; t_{1}=\varepsilon t ; t_{2}=\varepsilon^{2} t \\
\frac{\partial}{\partial x} \rightarrow \frac{\partial}{\partial x}+\varepsilon \frac{\partial}{\partial x_{1}}+\varepsilon^{2} \frac{\partial}{\partial x_{2}}+\cdots ; \frac{\partial}{\partial t} \rightarrow \frac{\partial}{\partial t}+\varepsilon \frac{\partial}{\partial t_{1}}+\varepsilon^{2} \frac{\partial}{\partial t_{2}}+\cdots
\end{gathered}
$$

As a result, the solution is represented in the form of a perturbation series such as,

$$
P=P_{0}\left(x, x_{1}, x_{2}, t, t_{1}, t_{2}\right)+\varepsilon P_{1}\left(x, x_{1}, x_{2}, t, t_{1}, t_{2}\right)+\varepsilon^{2} P_{2}\left(x, x_{1}, x_{2}, t, t_{1}, t_{2}\right)+\cdots,
$$

where $P_{0}, P_{1}$ and $P_{2}$ correspond to the above three scales of wave propagation and modification, respectively. It is important to note that high order terms $(>2)$ with regard to $\varepsilon$ from Equations (9) and (10) are neglected in the following analytical analysis under the assumption of a relatively small extent 
of non-uniformities of pipe diameters or cross-sectional areas. This assumption will be validated and discussed through numerical applications later in this study.

\section{Analytical Results and Analysis}

By applying the multi-scale perturbation method in Equation (9) to the transient wave equation in Equation (6), the analytical results of wave scattering with regard to the pressure wave envelopment and evolution can be obtained for both the regular case in Figure $4 \mathrm{~b}$ and the random case in Figure $4 \mathrm{a}$ of pipe diameter variations. For clarity and due to the page space limit, only the key steps and results are presented as follows, while the detailed derivations are neglected in this paper.

\subsection{Results of Regular Non-Uniformities}

For the analysis of the regular case of pipe diameter non-uniformities, it is assumed that the disordered pipe section has a regular variation magnitude of a pipe cross-section area as shown in Figure $4 \mathrm{~b}$, which is defined by the relatively disordered cross-sectional area (i.e., $\delta A=\triangle A / A_{0}$ ) following a periodic co-sinusoidal variation along the pipeline, as follows:

$$
A(x)=A_{0}\left(1+\varepsilon \delta A \cos \left(\lambda_{b} x\right)\right)=1+\varepsilon \delta A \cos \left(\lambda_{b} x\right),
$$

where $A_{0}$ is the mean value of the pipe cross-sectional area, assuming $A_{0}=1.0 \mathrm{~m}^{2}$ in this study for simplification; and $\lambda_{b}$ is the periodic length of pipe diameter disorders.

Based on the multi-scale wave perturbation technique, the wave scattering results in the regularly disordered pipeline can be obtained as three following cases:

1. Subcritical detuning: $0<\Omega<\Omega_{0}$,

$$
\left\{\begin{array}{l}
T\left(x_{1}\right)=\frac{\Omega \sinh K\left(L-x_{1}\right)+\mathrm{i} a K \cosh K\left(L-x_{1}\right)}{\Omega \sinh (K L)+\mathrm{i} a K \cosh (K L)} \\
R\left(x_{1}\right)=\frac{\Omega_{0} \sinh K\left(L-x_{1}\right)}{\Omega \sinh (K L)+\mathrm{i} a K \cosh (K L)}
\end{array},\right.
$$

where $T$ and $R$ are transmission and reflection coefficients respectively; $\Omega_{0}$ and $\Omega$ represent the incident wave frequency and pipe disorder variation frequency, respectively, and $\Omega_{0}=\delta A \omega / 2$, $\Omega=a \lambda_{b} ; x_{1}$ is the distance along the disordered section in the pipeline; $\mathrm{i}$ is the imaginary unit, and $i^{2}=-1 ; L$ is the total length of disordered section along the pipeline; and $K$ is the detuning (group) wave number and, $K=\sqrt{\left|\Omega_{0}^{2}-\Omega^{2}\right|} / a$.

2. Supercritical detuning: $\Omega>\Omega_{0}$,

$$
\left\{\begin{array}{l}
T\left(x_{1}\right)=\frac{\Omega \sin K\left(L-x_{1}\right)+\mathrm{i} a P \cos K\left(L-x_{1}\right)}{\Omega \sin (K L)+\mathrm{i} a K \cos (K L)} \\
R\left(x_{1}\right)=\frac{\Omega_{0} \sin K\left(L-x_{1}\right)}{\Omega \sin (K L)+\mathrm{i} a K \cos (K L)}
\end{array} .\right.
$$

3. Bragg resonance: $\Omega=\Omega_{0}$,

$$
\left\{\begin{array}{l}
T\left(x_{1}\right)=\frac{\cosh \Omega_{0}\left(L-x_{1}\right) / a}{\cosh \Omega_{0} L / a} \\
R\left(x_{1}\right)=-\mathrm{i} \frac{\sinh \Omega_{0}\left(L-x_{1}\right) / a}{\cosh \Omega_{0} L / a}
\end{array} .\right.
$$

Particularly, the Bragg resonance of the regularly disordered pipe in Equation (14) indicates that all waves are reflected completely by the disordered section along the pipeline. The analytical reflection coefficient $(R)$ along the disorder distance $\left(X=x_{1}\right)$ can be obtained according to Equation (14) and shown in Figure 5. The results of Figure 5 show clearly that, under a fixed disorder magnitude, the reflection coefficient $(R)$ is decreasing along the pipeline, which indicates that the wave perturbation energy is decayed gradually by the disorder section. As expected, the reflection coefficient $(R)$ at a fixed location of the pipe disorder section increases with the disorder magnitude 
$(\delta A)$ due to more wave energy reflecting back at the initial disorder section in the larger disorder magnitude $(\delta A)$ case. For example, the reflection coefficient at the starting location of the disorder section (i.e., $X / L_{0}=0$ ) could attain 0.9 when the disorder magnitude is about $10 \%$ of the mean value (i.e., $\varepsilon \sim 0.1$ ). Under this situation, there would be very little wave energy (perturbations) remaining at the end of pipe disorder section (since the total energy in the entire pipeline is conserved), resulting in a relatively high decrease-gradient of the reflection coefficient curve for larger $\delta A$ case as shown in Figure 5. More numerical validations to corroborate the analytical result are conducted later in this study.

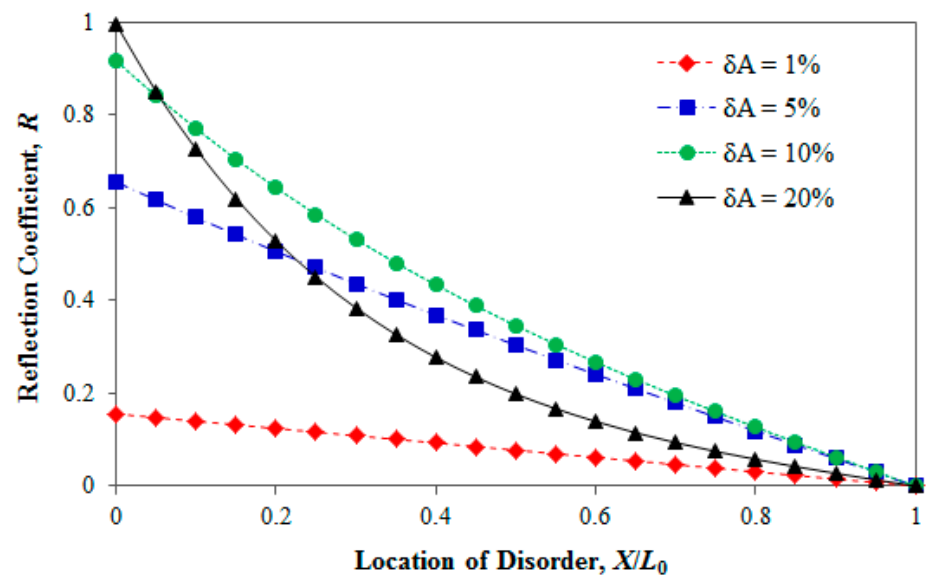

Figure 5. Analytical results of the reflection coefficient for the regular non-uniformities case.

\subsection{Results of Random Non-Uniformities}

For the case of random pipe diameter non-uniformities, as shown in Figure 4a, it is assumed that the pipe cross-sectional area follows the random variation function as,

$$
A(x)=A_{0}(1+\varepsilon \zeta(x))=(1+\varepsilon \zeta(x))
$$

where $\zeta(x)$ is a function that represents the random variation characteristics of the pipe cross-sectional area and is assumed to be of zero mean and be of standard deviation of $\sigma(x)$; other symbols are as defined above.

After applying the multi-scale perturbation analysis, the solution of the pressure wave envelopment to Equation (6) has the following form [4],

$$
B=B_{0} e^{-\lambda x}=B_{0} e^{-\lambda a t},
$$

where $B=B(x)=$ the amplitude of the wave envelope with distance or with the equivalent time $t=x / a$ with $a=$ wave speed along the pipe disorder section; $B_{0}=$ amplitude of the incident wave; $\lambda=\lambda_{r}-i \lambda_{i}$ is complex wave number, with $\lambda_{r}$ and $\lambda_{i}=$ wave damping factor and wave phase change (frequency shift) factor, respectively, and

$$
\lambda_{r}=\frac{\alpha k^{2} \sigma^{2}}{\alpha^{2}+4 k^{2}}, \lambda_{i}=-\frac{k \alpha^{2} \sigma^{2}}{2\left(\alpha^{2}+4 k^{2}\right)} .
$$

where $k=$ incident wave number and $k=\omega / a$, with $\omega=$ wave frequency; $\alpha=$ spatial correlation coefficient of the blockage and $\alpha \sim 1 / \lambda_{b}$ with $\lambda_{b}=$ correlation length which describes the spatial variability of pipe diameter non-uniformities.

The result of Equation (17) indicates that the wave amplitude exponentially decreases with longitudinal distance $(x)$. In other words, the wave is localized by the random non-uniformities of the 
pipe diameter or cross-sectional area along the pipeline. The localization distance can be defined and used for characterizing the wave scattering by the random diameter non-uniformities as

$$
L_{l o c}=\frac{1}{\varepsilon^{2} \lambda_{r}}=\frac{\alpha^{2}+4 k^{2}}{\varepsilon^{2} \sigma^{2} \alpha k^{2}}
$$

which represents the spatial distance for the wave amplitude decreased by an exponential factor of $e^{-1}$ as shown in Figure 6. This parameter $\left(L_{l o c}\right)$ is used later in this study for the evaluation of the wave scattering effect due to different pipe diameter non-uniformities.

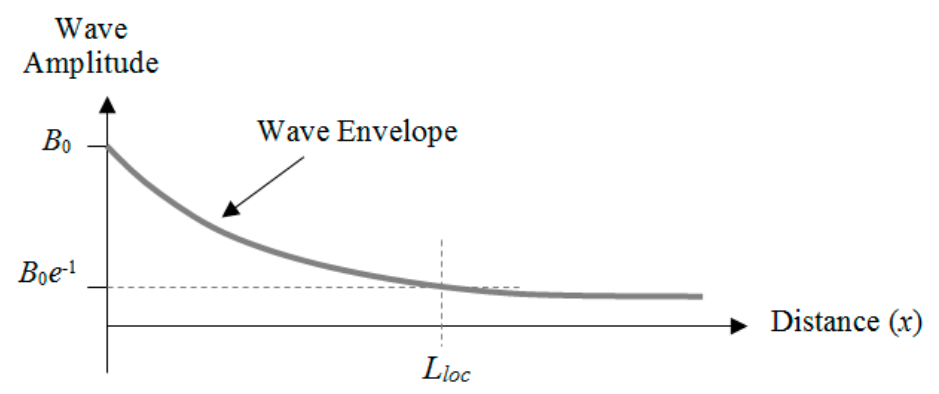

Figure 6. Wave localization by random pipe diameter non-uniformities.

Particularly, for a specified water supply pipeline under investigation, the correlation length of random pipe diameter non-uniformities (i.e., $L_{c o r} \sim 1 / \alpha$ ) is usually determinate (but maybe known or unknown for the analyst), and therefore, a dimensionless parameter (termed as wave scattering factor) can be further defined for better characterizing the wave scattering effect in that system as,

$$
\varphi=\alpha L_{l o c}=\alpha \frac{1}{\varepsilon^{2} \lambda_{r}}=\frac{\alpha^{2}+4 k^{2}}{\varepsilon^{2} \sigma^{2} k^{2}}=\frac{1}{\sigma_{A}^{2}}\left(4+\left(\frac{\alpha}{k}\right)^{2}\right) .
$$

Specifically, a smaller $\varphi$ value (shorter localized distance) means a relatively more significant wave scattering effect, and vice versa. Based on this result, the typical dependence relationship of the wave scattering factor on the properties of incident waves and pipe diameter non-uniformities can be shown in Figure 7. It is clearly shown in Figure 7 that when the incident wave length is around twice as long as the correlation length of the random pipe diameter non-uniformities (i.e., $k / \alpha=1 / 2$ ), the wave scattering effect would attain to maximum (i.e., minimum localized distance), and thus the incident wave can be strongly attenuated (scattered) along the pipe disorder section.

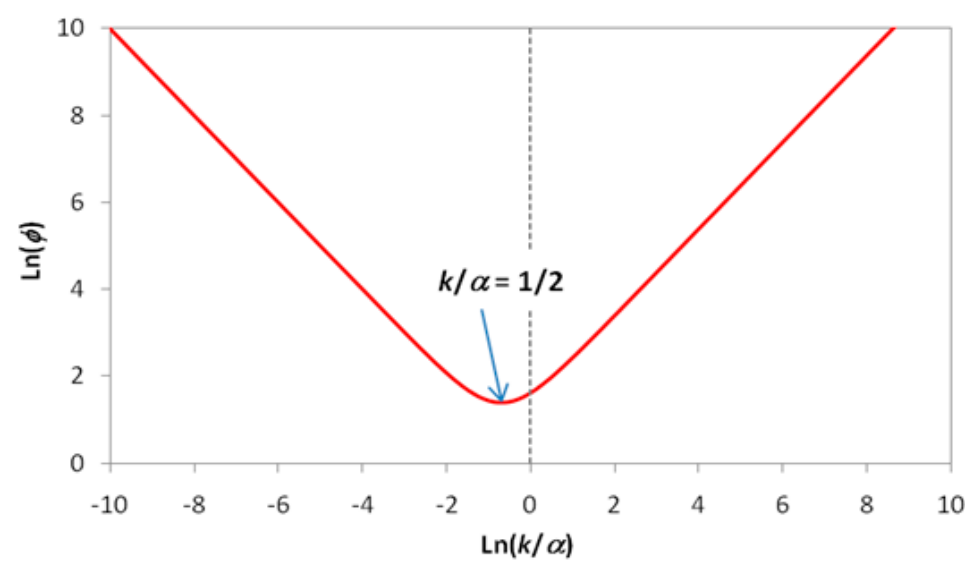

Figure 7. Dependence relationship of wave scattering factor on the incident waves and pipe diameter non-uniformities. 
It is necessary to point out that the obtained results of Equation (14) for the regular pipe disorder case and Equation (19) for the random pipe disorder case are obtained under the assumptions of linearization with relatively small extent of non-uniformities and no-reflection boundary conditions in the water pipeline system. The validity and accuracy of these results and assumptions are to be validated by the numerical simulations conducted later in this study.

\section{Numerical Validation}

\subsection{Settings of Numerical Tests}

To validate the derived analytical results presented above and evaluate the importance of the wave-scattering effect on transient wave propagation, the hypothetic pipeline system shown in Figure $8 \mathrm{a}$ is used for a numerical simulation, which consists of three pipe sections: upstream uniform pipe section, middle disordered pipe section (for testing), and downstream uniform pipe section. The length of each section is $2000 \mathrm{~m}$, and the no-reflection boundary condition from the two ends of the whole pipeline is imposed for the numerical simulation. For simplicity, all pipes are assumed to have a constant steady-state friction factor and wave speed (e.g., $f=0.01$ and $a=1000 \mathrm{~m} / \mathrm{s}$ ). The step approximation illustrated in Figure $4 \mathrm{c}$ is applied for the numerical simulation for both regular and random non-uniformities, which can be shown in Figure $8 b$.

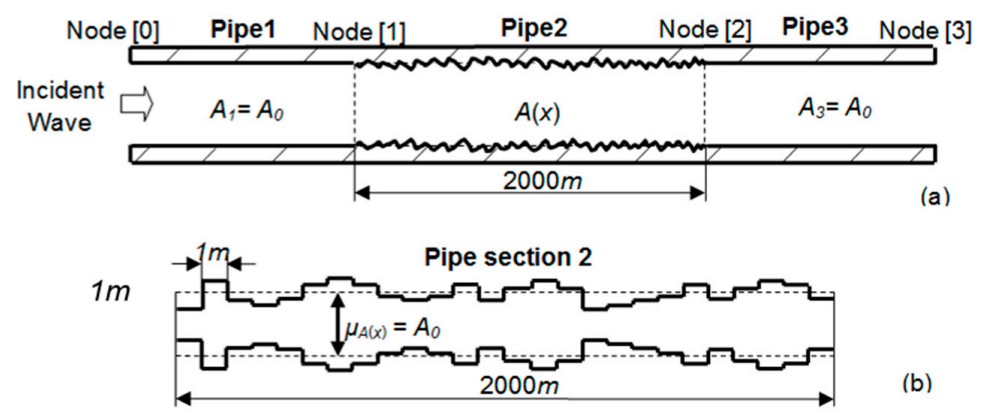

Figure 8. Schematic of numerical pipeline system: (a) three-section pipeline; (b) middle disorder section for testing.

A total of nine test cases, listed in Table 1, were conducted for numerical analysis, with cases 1 3 applied for a regular pipe disorder situation and cases $4 \sim 9$ for a random pipe disorder situation. Moreover, for each test, three different relationships between the incident wave length $\left(\lambda_{w}=1 / k\right)$ and the characteristic/correction length (distance) of pipe diameter non-uniformities $\left(\lambda_{b}=1 / \alpha\right)$ were considered for the evaluation. It is assumed that both types of non-uniformities (represented by pipe cross-sectional area) have a zero mean relative to the original nominal value. Note that $\lambda_{b}$ represents the periodic length of disordered diameters for the regular disorder case, while it represents the correlation length of disordered diameters in the pipeline for the random disordered cases (i.e., $1 / \alpha)$.

Table 1. Settings for the numerical test cases.

\begin{tabular}{|c|c|c|c|c|c|c|}
\hline Type & Case No. & $\lambda_{\mathrm{w}} / 2 \lambda_{b}$ & $A_{0}\left(\mathrm{~m}^{2}\right)$ & $\delta A$ & Distribution Function & Correlation Function \\
\hline \multirow{3}{*}{ Regular } & 1 & $>1$ & \multirow{3}{*}{1.0} & \multirow{3}{*}{$\sigma A / A_{0}=0.20$} & \multirow{3}{*}{$\begin{array}{c}\text { Degenerate } \\
\text { (deterministic) }\end{array}$} & \multirow{3}{*}{$\begin{array}{l}0 \text { for } \zeta \neq 0 \\
1 \text { for } \zeta=0\end{array}$} \\
\hline & 2 & $=1$ & & & & \\
\hline & 3 & $<1$ & & & & \\
\hline \multirow{6}{*}{ Random } & 4 & $>1$ & \multirow{3}{*}{1.0} & \multirow{3}{*}{$\sigma_{A} / A_{0}=0.23$} & \multirow{3}{*}{ Uniform } & \multirow{3}{*}{$e^{-\alpha|\zeta|}$} \\
\hline & 5 & $=1$ & & & & \\
\hline & 6 & $<1$ & & & & \\
\hline & 7 & $>1$ & \multirow{3}{*}{1.0} & \multirow{3}{*}{$\sigma_{A} / A_{0}=0.23$} & \multirow{3}{*}{ Upper triangular } & \multirow{3}{*}{$e^{-\alpha|\zeta|}$} \\
\hline & 8 & $=1$ & & & & \\
\hline & 9 & $<1$ & & & & \\
\hline
\end{tabular}


Initially, the pipeline system was considered to be under a steady state. Note that for comparison, the results for the completely uniform pipeline without non-uniformities (termed as the "intact" case hereafter) were also obtained for each test case. For transient generation, the incident wave at the upstream entrance of the pipeline was assumed to be a sinusoidal perturbation of pressure head signal as:

$$
H(t)=H_{0}\left[1+R_{f} \sin (\omega t)\right]
$$

where $H=$ instant pressure head; $H_{0}=$ initial steady pressure head level; $R_{f}=$ amplitude factor of incident wave and $R_{f}=0.2$ in this study; and $\omega=$ incident wave frequency.

For test cases of the random pipe diameter non-uniformities, two kinds of probability distributions were considered for the randomness of the non-uniformities: one followed uniform distribution (for cases 4 6) and the other was upper triangular distribution (for cases 7 9). It was assumed that the random variables of pipe diameter disorder were correlated with an exponential function along the pipeline in the spatial domain. Other numerical settings for different cases are listed in Table 1.

\subsection{Validation for Regular Case}

In the regular disordered tests, the total disordered distance was assumed to be $2000 \mathrm{~m}\left(L_{0}\right)$ and there were a total of 20 uniformly spaced reaches with each $100 \mathrm{~m}\left(\lambda_{b}=200 \mathrm{~m}\right)$. A continuously sinusoidal incident wave defined by Equation (20) was used in this study and the incident wave frequency was adjusted to achieve the three cases: $\lambda_{w} / 2 \lambda_{b}>1, \lambda_{w} / 2 \lambda_{b}=1$ and $\lambda_{w} / 2 \lambda_{b}<1$. The envelope of the maximum and minimum pressure head profiles along the disordered pipe section was extracted from the numerical results and plotted in Figure 9a-c.
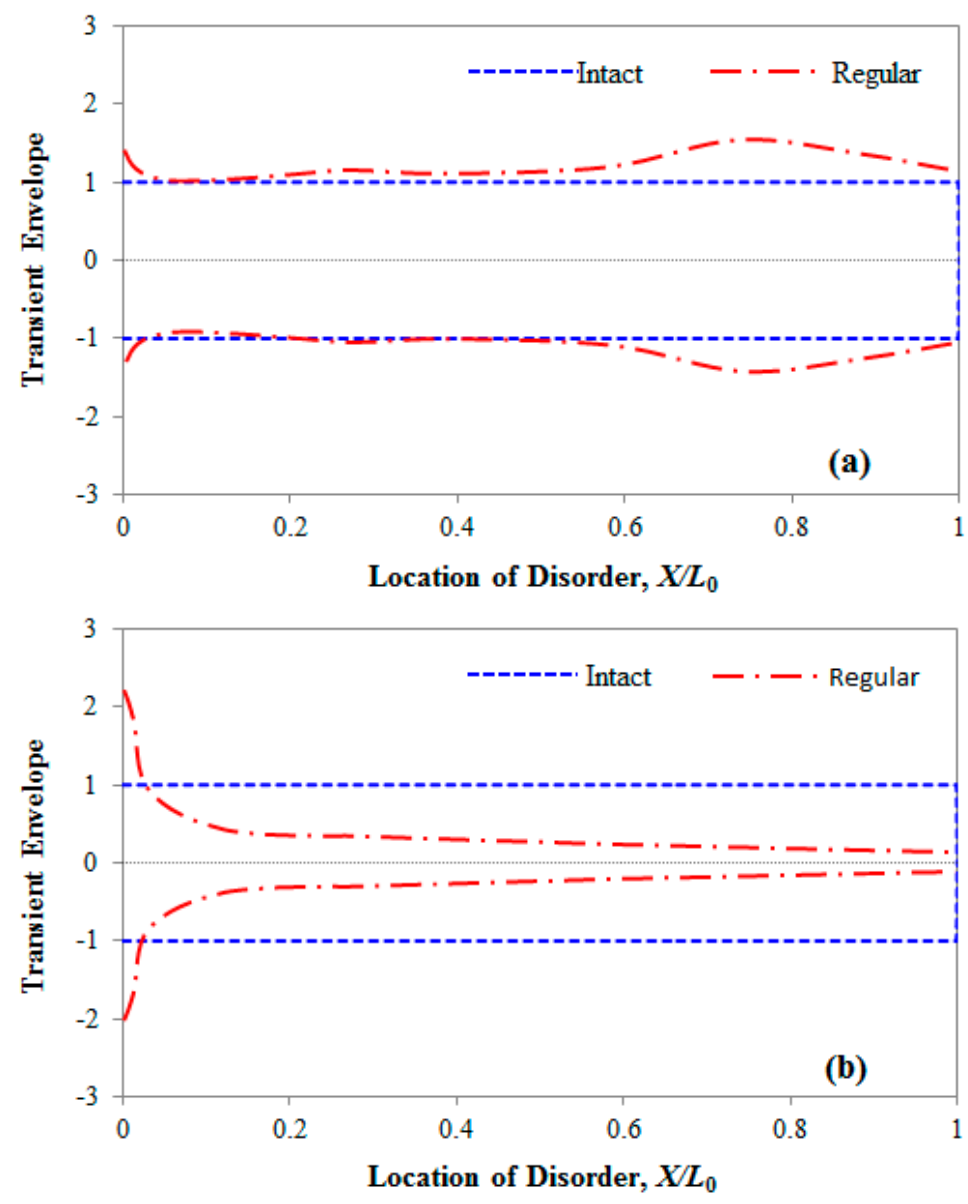

Figure 9. Cont. 


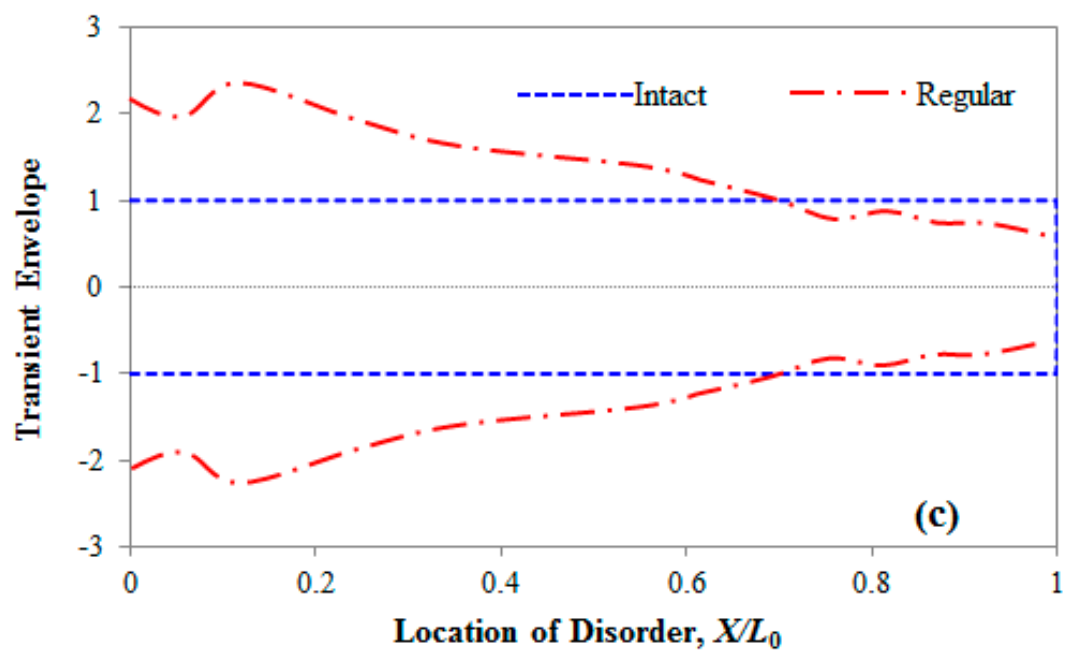

Figure 9. Results of the pressure wave envelope for regular disorder cases: (a) $\lambda_{w} / 2 \lambda_{b}=1$ and $\delta A=0.05$; (b) $\lambda_{w} / 2 \lambda_{b}=1$ and $\delta A=0.10$; and (c) $\lambda_{w} / 2 \lambda_{b}=1$ and $\delta A=0.20$.

The results comparison in Figure 9 indicates that the attenuation of the pressure wave along the pipeline due to the wave-scattering effect behaves much more significantly for case two, with $\lambda_{w} / 2 \lambda_{b}=1$ in Figure $9 \mathrm{~b}$, than other two cases, $\left(\lambda_{w} / 2 \lambda_{b}>1\right.$ in Figure $9 \mathrm{a}$ and $\lambda_{w} / 2 \lambda_{b}<1$ in Figure $9 \mathrm{c}$, which is consistent with the analytical results of Equations (12)-(14) and Figure 7. Meanwhile, for the cases of $\lambda_{w} / 2 \lambda_{b}>1$ and $\lambda_{w} / 2 \lambda_{b}<1$, the pressure wave envelopes were larger than that of the intact case because of the superposition of the scattered waves. To further validate the analytical solution, the reflection coefficients $(R)$ of perfect resonance for case no. two were calculated and plotted in Figure 10. As shown in Figure 10, obvious discrepancies were observed between the analytical and numerical results, which were actually increasing with the disorder magnitude $(\delta A)$. This result implies that the linearized analytical solution can provide good estimations for the wave scattering effect of a relatively small pipe disorder situation, which is due to the linearization assumption imposed by the analytical analysis.

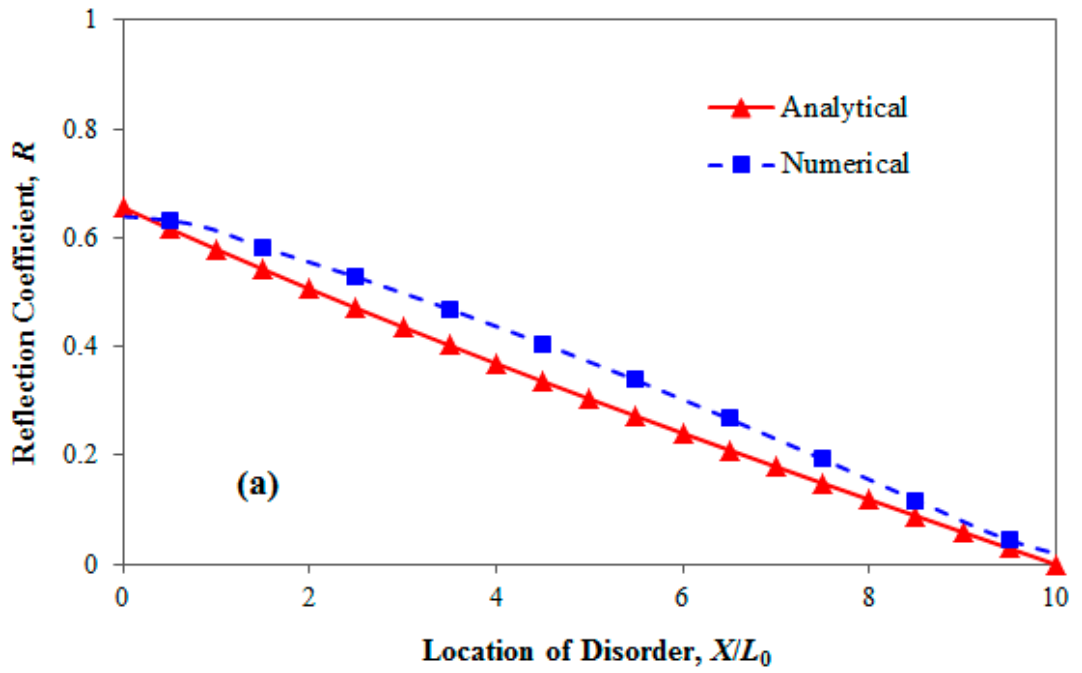

Figure 10. Cont. 

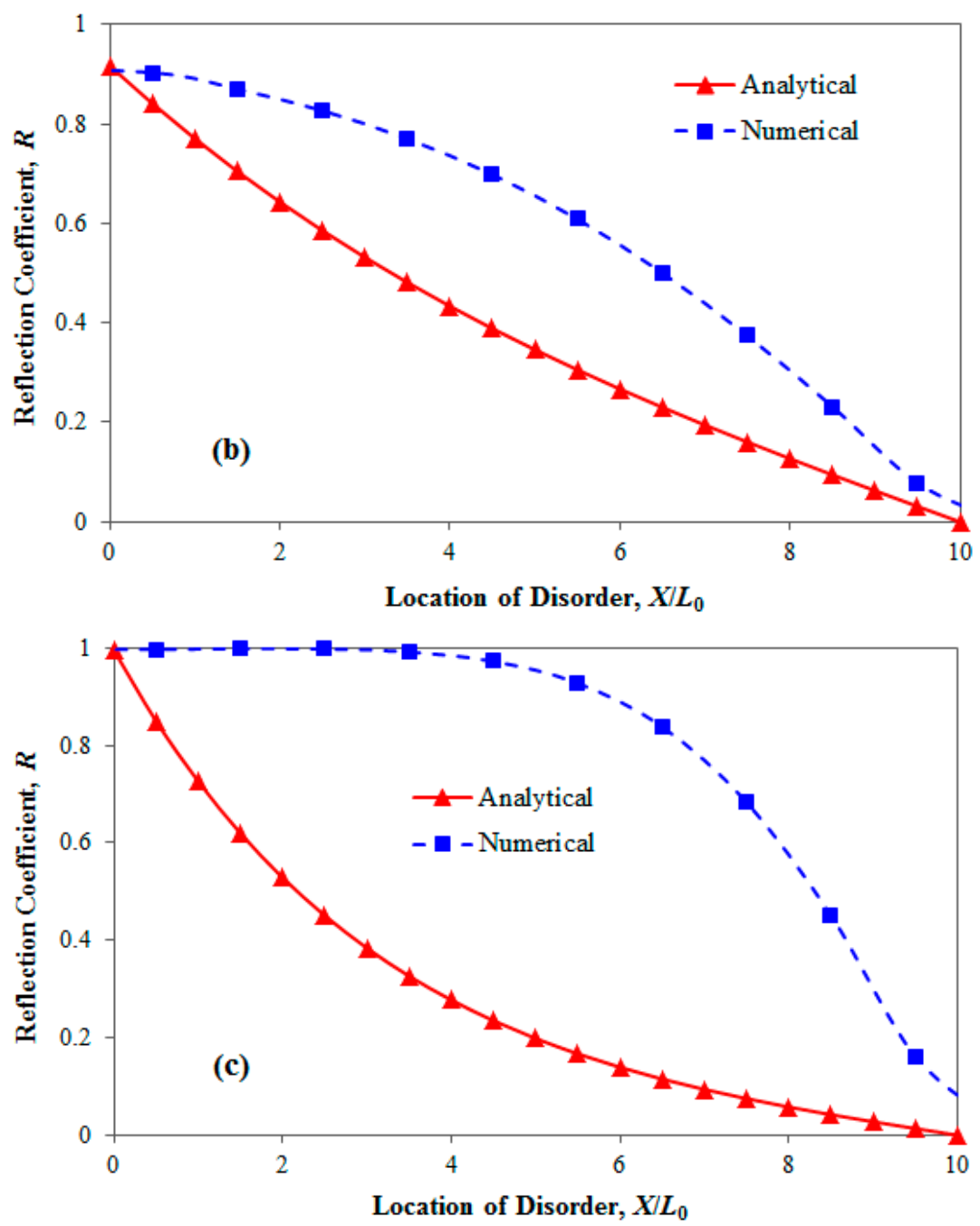

Figure 10. Comparisons of the numerical and analytical results of the reflection coefficients for the case of $\lambda_{w} / 2 \lambda_{b}=1$ : (a) $\delta A=0.05$; (b) $\delta A=0.10$; and (c) $\delta A=0.20$.

\subsection{Validation for the Random Case}

In the numerical tests involving randomly disordered pipes, the disordered diameters were assumed to be spatially correlated along the pipeline. For simulations, it was assumed that the continuously-correlated random diameters could be discretized into many small reaches, with each reach $1 \mathrm{~m}$ representing one spatial random point of the original continuous disordered section. In this study, the generation of samples of randomly correlated diameters was based on the "NORTA" (normal to anything) theorem, which was developed by Ghosh [12]. Thereafter, a Monte-Carlo simulation (MCS) with 500 samples was conducted and the statistical results were retrieved for the analysis [13].

With the MCS-based numerical simulations, the pressure wave profiles were obtained and shown in Figures 11-13 for the cases of $\lambda_{w} / 2 \lambda_{b}>1, \lambda_{w} / 2 \lambda_{b}<1$ and $\lambda_{w} / 2 \lambda_{b}=1$, respectively. It is clear from these results that the pressure wave amplitude decays exponentially with distance along the pipe with random diameter non-uniformities. Moreover, the results for both uniform and triangular distributions of random non-uniformities indicate that the wave scattering effect behaves most significantly when $\lambda_{w} / 2 \lambda_{b}=1$ (see Figure 12), which is similar to the results of the regular disorder cases analyzed above. The results also imply that the different probability distributions (uniform or triangular) for random non-uniformities along the pipeline have little impact on the wave-scattering effect, as long as the other parameters remain the same, e.g., mean, standard deviation and correlation. 
For validation, the analytical and numerical results of dimensionless localization length $(\varphi)$ in Equation (19) for different cases and their relative errors were calculated and are listed in Table 2. The results show that the maximum relative error is less than $5 \%$, which implies good prediction by the linear analytical solutions of Equation (19) for wave scattering in random pipe disorder cases. On this point, the analytical results of Equation (19) have been validated for describing the qualitative influence and importance trend of wave scattering induced by random diameter non-uniformities in the pipe.

Table 2. Analytical and numerical results of the wave scattering factor.

\begin{tabular}{cccccccc}
\hline \multirow{2}{*}{ Case No. } & \multicolumn{3}{c}{ Uniform Distribution } & \multicolumn{3}{c}{ Upper Triangular Distribution } \\
\cline { 3 - 8 } & & $\mathbf{4}$ & $\mathbf{5}$ & $\mathbf{6}$ & $\mathbf{7}$ & $\mathbf{8}$ & $\mathbf{9}$ \\
\hline \multirow{2}{*}{ Wave scattering } & Analytical & 125.0 & 75.0 & 159.4 & 125.0 & 75.0 & 159.4 \\
factor $(\varphi)$ & Numerical & 127.5 & 74.5 & 157.0 & 125.8 & 76.3 & 152.5 \\
& Relative error $(\%)$ & 2.0 & 0.7 & 1.5 & 0.7 & 1.7 & 4.5 \\
\hline
\end{tabular}
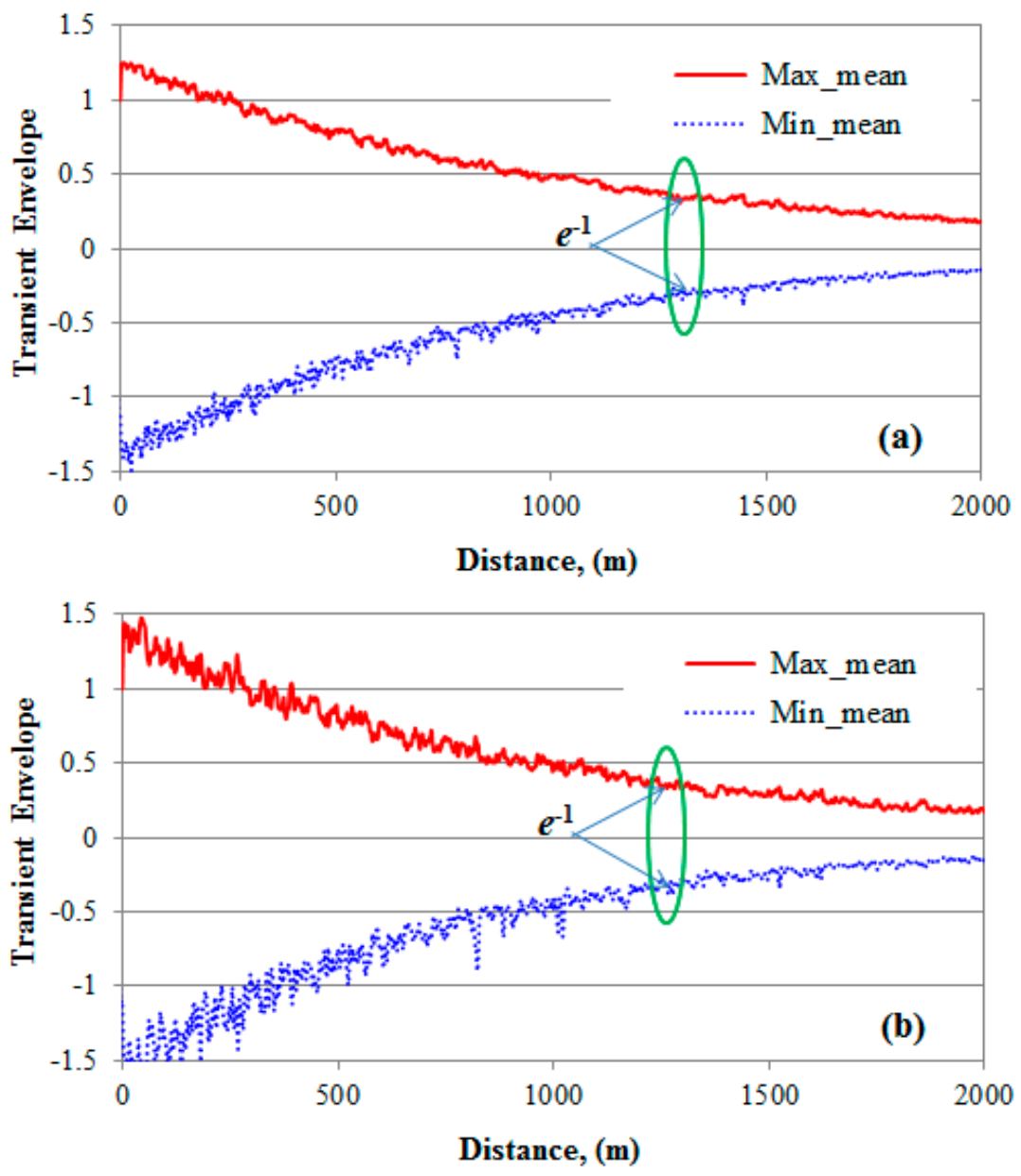

Figure 11. Results of the random disorder case with $\lambda_{w} / 2 \lambda_{b}>1$ : (a) uniform distribution and $\alpha=0.6 \mathrm{~m}^{-1} ;(\mathbf{b})$ triangular distribution and $\alpha=0.6 \mathrm{~m}^{-1}$. 

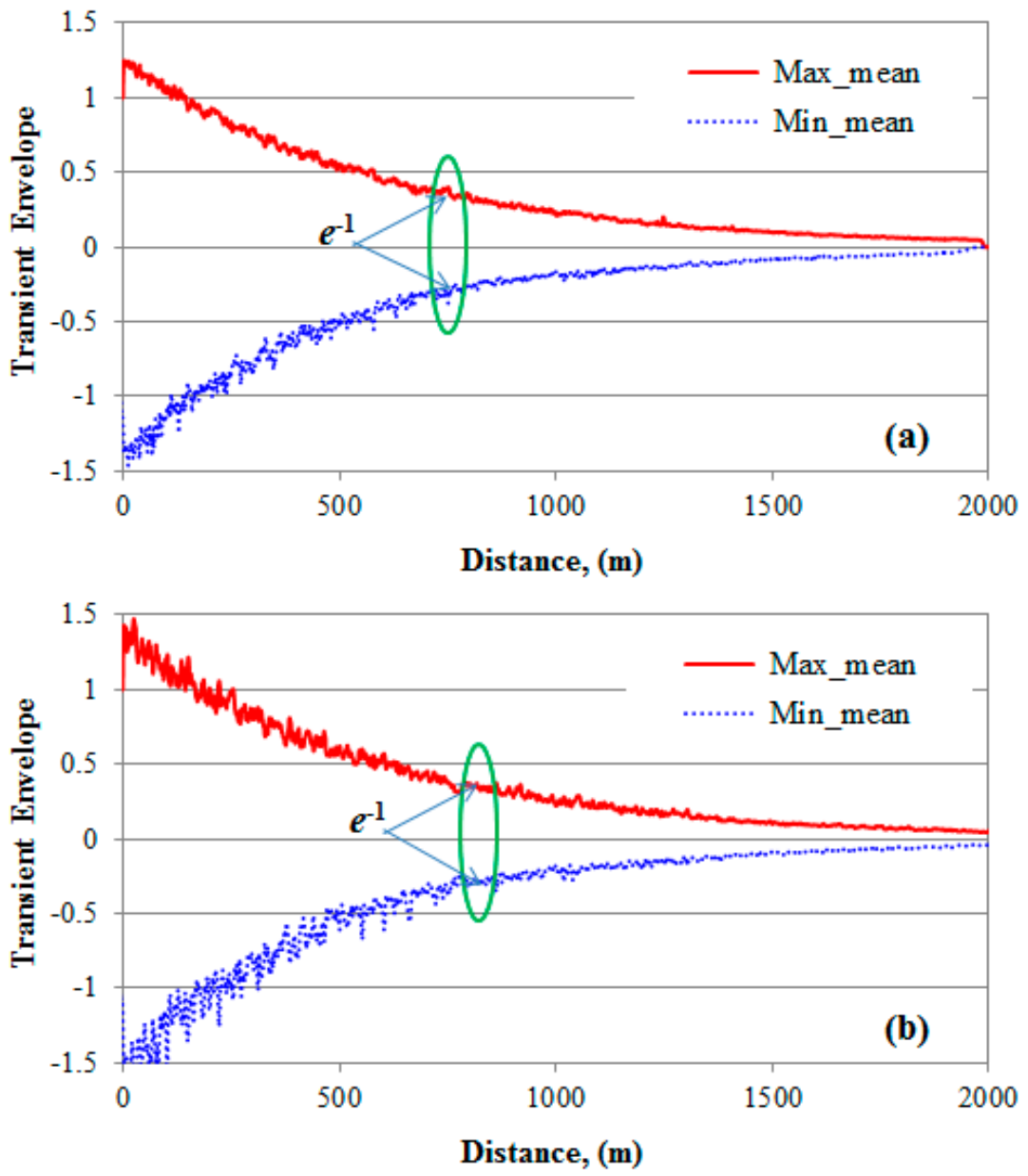

Figure 12. Results of the random disorder case with $\lambda_{w} / 2 \lambda_{b}=1$ : (a) uniform distribution and $\alpha=0.2 \mathrm{~m}^{-1} ;(\mathbf{b})$ triangular distribution and $\alpha=0.2 \mathrm{~m}^{-1}$.

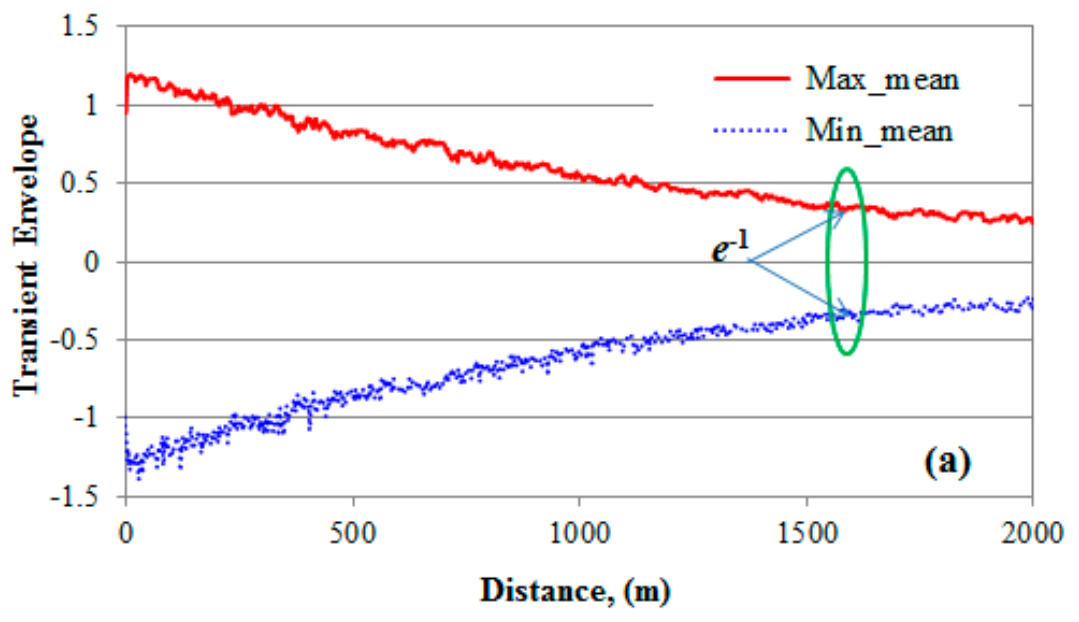

Figure 13. Cont. 


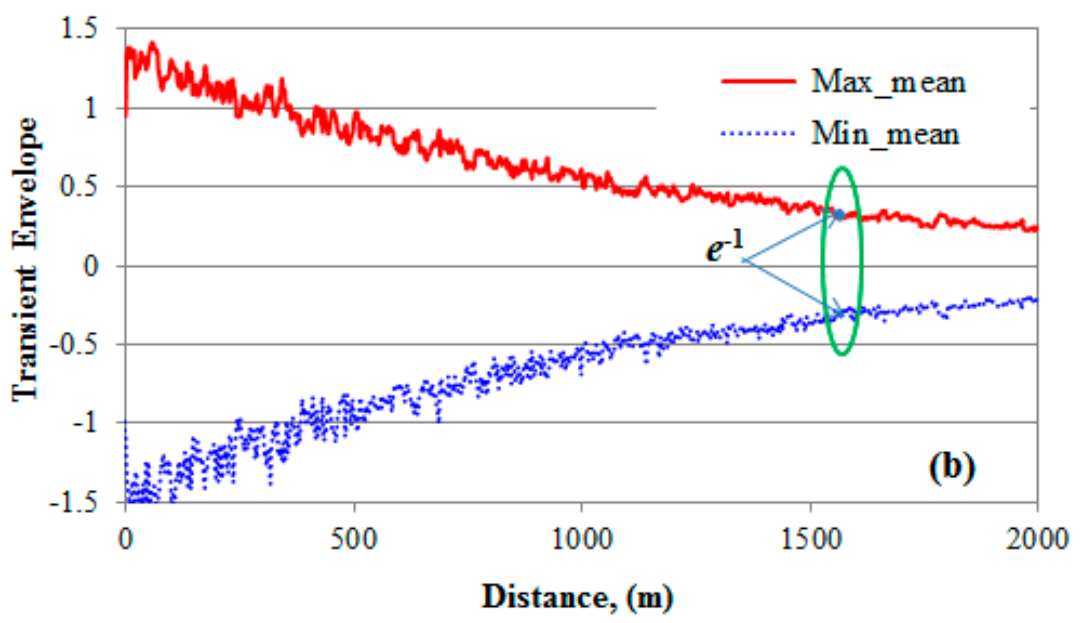

Figure 13. Results of the random disorder case with $\lambda_{w} / 2 \lambda_{b}<1$ : (a) uniform distribution and $\alpha=0.05 \mathrm{~m}^{-1} ;(\mathbf{b})$ triangular distribution and $\alpha=0.05 \mathrm{~m}^{-1}$.

\section{Results Discussion and Implications}

\subsection{Energy Analysis of Transient Wave Scattering}

It is clear in Equation (19) that the localization distance (by wave scattering factor $\varphi$ ) decreases with an increase of the amplitude of pipe random non-uniformities $\left(\sigma_{A}\right)$ because of more serious reflections by these non-uniformities in the pipeline. To further explain and understand the wave scattering effect, an energy analysis is performed based on the energy formulations in previous studies $[14,15]$. The results of case no. four in Table 2 are retrieved from the model and plotted in Figure 14. It is clearly shown in Figure 14 that the total energy in the pipeline system with random variation in diameters is always conserved, although each form of the energy (kinetic or internal) changes significantly with time. In other words, as a result of the wave scattering effect, the total energy has been re-distributed in the system due to the pipe diameter non-uniformities such that pressure wave envelopment is scattered significantly along the pipeline as indicated in the analytical solutions.

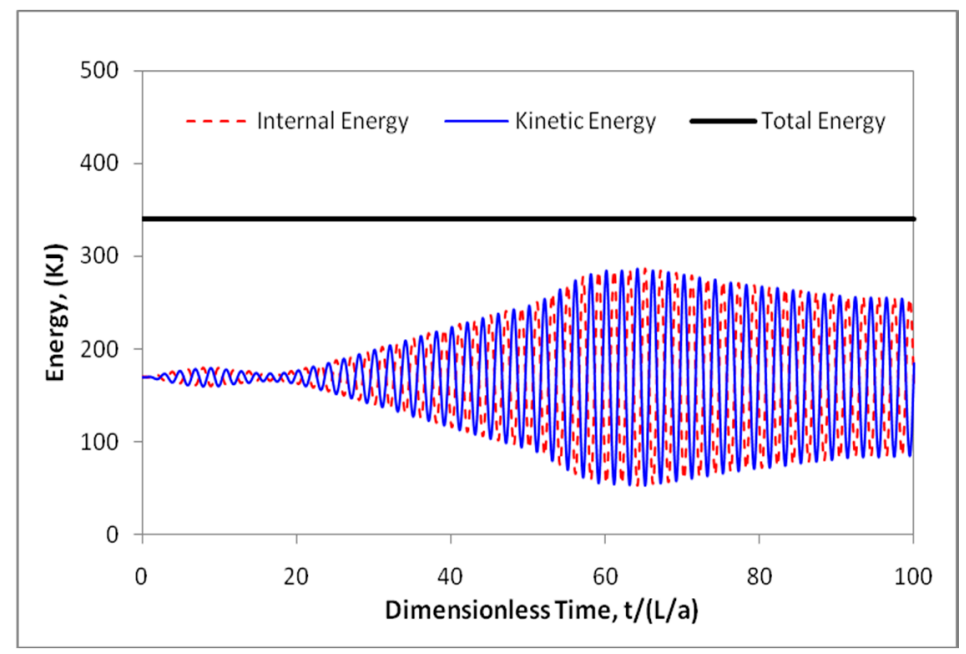

Figure 14. Energy results of transient wave scattering along the pipeline.

\subsection{Impacts on Transient Modelling and Analysis}

The pressure wave envelopment attenuation in the present waterhammer models is usually attributed to friction damping (and viscoelasticity damping if pipes are plastic) $[9,15]$. With the 
existence of wave scattering effects, especially in the aged pipeline system, the actual damping of pressure waves might not be fully represented by only the friction (and pipe-wall viscoelasticity if appropriate), while the wave scattering effect with energy re-distribution in the system may provide a great contribution to the total attenuation. Therefore, it is necessary to inspect the relative importance of wave scattering and friction and their roles in pressure wave envelopment attenuation, such that the transient flow behaviors in these disordered pipes can be well understood and accurately simulated by waterhammer models.

In this regard, the analytical results derived above in this study may provide useful guidance and estimation of the influence of wave scattering. Specifically, the analytical results of Equation (19) in this study indicates an increasing localization distance $(\varphi)$ and thus a decreasing wave scattering effect with an increase of the ratio of $\alpha / k$. That is, when the incident wave length is shorter in comparison with the correlation length of random diameter non-uniformities (i.e., $\alpha<<k$ ), the wave scattering effect becomes more significant and the waves are localized more seriously in the pipeline. As a result, high frequency waves propagating in the randomly disordered pipeline can be greatly scattered such that most of the waves are reflected back upstream (i.e., the incident part) and hardly transmitted downstream (i.e., the outgoing part), if only the pipeline is long enough.

In practical transient (waterhammer) systems, however, the incident waves are usually "fast and sharp" signals, for example caused by a sudden closure or opening of valves, the starting or stopping of pumps, etc., where the operation time duration is rather short. From this perspective, in pipe systems with potential random diameter non-uniformities and under transient conditions, the wave scattering induced wave envelopment attenuation is generally dominant in comparison with friction damping. In this regard, many typical examples have been shown in previous studies $[4,5,8]$, where significant discrepancies were commonly observed between the real data (from both laboratory and field tests) and the numerical model results (e.g., MOC-based simulation with steady and/or unsteady friction components).

Another important implication for transient system analysis is that the random non-uniformities can cause an increase in the pressure head (also energy) in certain regions of the pipeline system since most of the waves are reflected or trapped by the disordered section of the pipeline. This is clearly worrisome for aged pipes since such a pressure increase was likely not accounted for when pipes were designed for the water supply system. Therefore, the wave behavior in aged pipelines might become very complicated due to the potential wave scattering effect, such that the design schemes of system strength from their initial new states may become overestimated or underestimated for some sections of the pipe system.

\subsection{Impacts on Transient-Based Leak Detection}

Transient-based defect detection techniques are being developed by various researchers [16-26]. The idea is to intentionally inject a wave, typically a pressure variation by, for example, changing a valve setting, and then measure the subsequent pressure response of the system. The key is to find the signature of the defect in the measured signal and use it to identify the nature, location and size of the defect.

A previous study by the author [27] has demonstrated that the current transient-based pipe defect detection methods are mainly dependent on wave damping and reflections and that the "fast and sharp" input wave signals are preferable to these methods. Clearly, such approaches could become intractable in the presence of random non-uniformities in the pipeline system. The non-uniformities in pipes may be due to pipe diameter, material and thickness as well as fluid properties such the case of pumped sewerage. Consequently, these pipe defect detection methods are particularly difficult to be applied in the aged pipes and/or sewage drainage systems where potential non-uniformities commonly exist. For illustration, two test cases (T1 and T2) listed in Table 3 are examined in which the disordered pipe diameter shown in Figure 8 is present. Four types of leak detection methods-transient 
reflection method (TRM), transient damping method (TDM), system response function method (SRFM), and inverse transient method (ITM) - were used in this investigation [27].

Table 3. Results of leak detection under the presence of wave scattering in the pipeline.

\begin{tabular}{ccccccc}
\hline \multirow{2}{*}{ Case } & $\begin{array}{c}\text { Real Leak Information, } \\
x_{L}{ }^{*} \& A_{L}{ }^{*}\end{array}$ & \multicolumn{4}{c}{ Predicted Leak Information, $x_{p}{ }^{*} \& A_{p}^{*}$} & \multirow{2}{*}{$\begin{array}{c}\text { Max. Error, } \mid x_{L}{ }^{*}- \\
x_{p}^{*} \mid\end{array}$ \& $\left|A_{L}{ }^{*}-A_{p}^{*}\right|$} \\
\cline { 3 - 6 } & No Leak & $0.50 \& 0.01$ & $0.50 \& 0.038$ & $0.25 \& 0.024$ & $0.46 \& 0.031$ & $-\&-$ \\
\hline T1 & $0.1 \& 0.002$ & $0.49 \& 0.012$ & $0.44 \& 0.042$ & $0.17 \& 0.019$ & $0.34 \& 0.035$ & $39 \% \& 40 \%$ \\
\hline T2 & & & &
\end{tabular}

In Table 3, leak location and size $\left(x_{L}{ }^{*} \& A_{L}{ }^{*}\right)$ were normalized by the total pipe length and average pipe cross-sectional area, respectively. The relative errors of predicted dimensionless leak location by using these four methods are also listed in the table. The results of case T1 show that the additional pseudo leak is detected by all four methods, while actually there is no leak along the pipeline. In case T2, the maximum predicted error using the four methods can reach $39 \%$ and $40 \%$ for the leak location and size, respectively. This also indicates that the four leakage detection methods are invalid or inaccurate when wave scattering induced reflections and "damping" exist.

Actually, many recent studies have evidenced the wave scattering phenomenon in water supply distribution systems, where the water supply demand (and thus the pressure head) was observed to pulse frequently and continuously, although transient oscillations were relatively small [28-33]. Consequently, these preliminary results and analysis indicated that the wave scattering effect could have a great influence on both transient system analysis and transient-based utilization in urban water pipeline systems. More attention needs to be paid to the impact of the wave scattering effect on transient wave behavior and propagation (reflection and damping) so that present models and techniques can be applied with confidence to practical pipeline systems.

\section{Conclusions}

The analytical expressions for transient wave evolution in water pipelines with different non-uniformities were derived in this paper by using the multi-scale wave perturbation method, which was validated and examined through extensive 1D numerical simulations. The analytical and numerical results showed the fact that pressure waves are attenuated significantly by both the regular and random pipe diameter non-uniformities along the longitudinal direction, which has been widely observed in the numerical and experimental results in the literature. Meanwhile, the derived results imply that the importance and influence of the wave scattering effect in the pipeline is dependent on the relationship between the incident wave frequency and non-uniform pipe diameter variation frequency. Particularly, the wave scattering induced wave localization length becomes smaller and thus the attenuation of wave envelope is more significant when the ratio of incident wave length and the correlation length of the non-uniformities becomes smaller. As a result, for the specific pipeline system with the existence of pipe diameter non-uniformities, the wave scattering effect becomes critical for the high frequency incident waves, which is, however, common in water hammer flows.

The preliminary results and findings of this study are useful and implicative to both transient theory (transient modelling and analysis) and practice (transient utilization). Firstly, the wave behavior in the aged pipelines might become very complicated due to the unavoidable wave scattering effect such that the design schemes of the system strength from their initial new states may become invalid/inaccurate (overestimated or underestimated) for the regional or global pipe system. Secondly, the complicated wave reflections and amplitude attenuation induced by the wave scattering effect may result in inaccurate predictions or even the invalidity of current transient-based pipe defect detection methods. Finally, but not least importantly, the transient (waterhammer) flow theories, such as friction and viscoelasticity models, which are usually validated and calibrated through the measured data of pressure wave attenuation and reflections from practical systems, may be wrongly 
represented and explained if the potential wave scattering effect has not been considered or not been well included in the analysis.

It is important to note that the assumption of a relative small extent of pipe diameter non-uniformities has been used in the analytical analysis of this study, where high-order $(>2)$ terms were ignored in the derivation process. With this assumption, clear discrepancies, especially for the regular case of pipe non-uniformities, were observed between the analytical and numerical results obtained in this study. From this perspective, more future work is required to further validate and verify the accuracy and applicability of the derived analytical results in this paper.

Acknowledgments: This research work was supported by (1) the Hong Kong Research Grants Council (RGC) under the projects No. 25200616, No. 15201017 and No. 3-RBAB; (2) the Hong Kong Polytechnic University (PolyU) under projects No. 1-ZVCD and No. 1-ZVGF.

Conflicts of Interest: The author declares no conflict of interest.

\section{References}

1. Dolan, M.; Dean, R.G. Multiple longshore sand bars in the Upper Chesapeake Bay. Estuar. Coast. Shelf Sci. 1985, 21, 721-743. [CrossRef]

2. Belzons, M.; Guazzelli, E.; Parodi, O. Gravity waves on a rough bottom: Experimental evidence of one-dimensional localization. J. Fluid Mech. 1988, 186, 539-558. [CrossRef]

3. Devillard, P.; Dunlop, F.; Souvillard, B. Localization of gravity waves on a channel with random bottom. J. Fluid Mech. 1988, 186, 521-538. [CrossRef]

4. Duan, H.F.; Lee, P.J.; Che, T.C.; Ghidaoui, M.S.; Karney, B.W.; Kolyshkin, A.A. The influence of non-uniform blockages on transient wave behavior and blockage detection in pressurized water pipelines. J. Hydro-Environ. Res. 2017, 17, 1-7. [CrossRef]

5. Duan, H.F.; Lee, P.J.; Tuck, J. Experimental investigation of wave scattering effect of pipe blockages on transient analysis. Procedia Eng. 2014, 89, 1314-1320. [CrossRef]

6. Anderson, P.A. Absence of diffusion in certain random lattices. Phys. Rev. 1958, 109, 1492-1505. [CrossRef]

7. Mei, C.C.; Stiassnie, M.; Yue, D.K.P. Theory and Applications of Ocean Surface Waves, Part 1: Linear Aspects; World Scientific: Singapore, 2005.

8. McInnis, D.; Karney, B.W. Transients in distribution networks: Field tests and demand models. J. Hydraul. Eng. 1995, 121, 218-231. [CrossRef]

9. Ghidaoui, M.S.; Zhao, M.; McInnis, D.A.; Axworthy, D.H. A review of waterhammer theory and practice. Appl. Mech. Rev. 2005, 58, 49-76. [CrossRef]

10. Wylie, E.B.; Streeter, V.L.; Suo, L. Fluid Transients in Systems; Prentice Hall, Inc.: Englewood Cliffs, NJ, USA, 1993.

11. Nayfeh, A.H. Introduction to Perturbation Techniques; John Wiley \& Sons: New York, NY, USA, 1981.

12. Ghosh, S. Dependence in Stochastic Simulation Models. Ph.D. Thesis, Cornell University, New York, NY, USA, 2004.

13. Tung, Y.K.; Yen, B.C.; Melching, C.S. Hydrosystems Engineering Reliability Assessment and Risk Analysis; McGraw-Hill Company, Inc.: New York, NY, USA, 2006.

14. Karney, B.W. Energy relations in transient closed-conduit flow. J. Hydraul. Eng. 1990, 116, 1180-1196. [CrossRef]

15. Duan, H.F.; Ghidaoui, M.S.; Lee, P.J.; Tung, Y.K. Unsteady friction and visco-elasticity in pipe fluid transients. J. Hydraul. Res. 2010, 48, 354-362. [CrossRef]

16. Brunone, B. Transient test-based technique for leak detection in outfall pipes. J. Water Resour. Plan. Manag. 1999, 125, 302-306. [CrossRef]

17. Sun, J.L.; Wang, R.; Duan, H.F. Multiple-fault detection in water pipelines using transient time-frequency analysis. J. Hydroinform. 2016, 18, 975-989. [CrossRef]

18. Wang, X.J.; Lambert, M.F.; Simpson, A.R.; Liggett, J.A.; Vítkovský, J.P. Leak detection in pipeline systems using the damping of fluid transients. J. Hydraul. Eng. 2002, 128, 697-711. [CrossRef]

19. Duan, H.F.; Lee, P.J.; Ghidaoui, M.S.; Tung, Y.K. Extended blockage detection in pipelines by using the system frequency response analysis. J. Water Resour. Plan. Manag. 2011, 138, 55-62. [CrossRef] 
20. Duan, H.F.; Lee, P.J.; Ghidaoui, M.S.; Tung, Y.K. Leak detection in complex series pipelines by using system frequency response method. J. Hydraul. Res. 2011, 49, 213-221. [CrossRef]

21. Duan, H.F.; Lee, P.J. Transient-based frequency domain method for dead-end side branch detection in reservoir-pipeline-valve systems. J. Hydraul. Eng. 2016, 142, 04015042. [CrossRef]

22. Ferrante, M.; Brunone, B. Pipe system diagnosis and leak detection by unsteady-state tests-1: Harmonic analysis. Adv. Water Resour. 2003, 26, 95-105. [CrossRef]

23. Lee, P.J.; Lambert, M.F.; Simpson, A.R.; Vítkovský, J.P.; Liggett, J. Experimental verification of the frequency response method for pipeline leak detection. J. Hydraul. Res. 2006, 44, 693-707. [CrossRef]

24. Meniconi, S.; Duan, H.F.; Lee, P.J.; Brunone, B.; Ghidaoui, M.S.; Ferrante, M. Experimental investigation of coupled frequency and time-domain transient test-based techniques for partial blockage detection in pipes. J. Hydraul. Eng. 2013, 139, 1033-1040. [CrossRef]

25. Kim, S. Impedance method for abnormality detection of a branched pipeline system. Water Resour. Manag. 2016, 30, 1101-1115. [CrossRef]

26. Sattar, A.M.; Chaudhry, M.H. Leak detection in pipelines by frequency response method. J. Hydraul. Res. 2008, 46, 138-151. [CrossRef]

27. Duan, H.F.; Lee, P.J.; Ghidaoui, M.S.; Tung, Y.K. Essential system response information for transient-based leak detection methods. J. Hydraul. Res. 2010, 48, 650-657. [CrossRef]

28. Stephens, M.L. Transient Response Analysis for Fault Detection and Pipeline Wall Condition Assessment in Field Water Transmission and Distribution Pipelines and Networks. Ph.D. Thesis, The University of Adelaide, Adelaide, Australia, 2008.

29. Duan, H.F. Investigation of Factors Affecting Transient Pressure Wave Propagation and Implications to Transient Based Leak Detection Methods in Pipeline Systems. Ph.D. Thesis, The Hong Kong University of Science and Technology, Hong Kong, China, 2011.

30. Blokker, E.J.M.; Vreeburg, J.H.G.; van Dijk, J.C. Simulating residential water demand with a stochastic end-use model. J. Water Resour. Plan. Manag. 2010, 136, 19-26. [CrossRef]

31. Creaco, E.; Campisano, A.; Franchini, M.; Modica, C. Unsteady flow modeling of pressure real-time control in water distribution networks. J. Water Resour. Plan. Manag. 2017, 143, 04017056. [CrossRef]

32. Creaco, E.; Pezzinga, G.; Savic, D. On the choice of the demand and hydraulic modeling approach to WDN real-time simulation. Water Resour. Res. 2017, 53, 6159-6177. [CrossRef]

33. Buchberger, S.G.; Carter, J.T.; Lee, Y.H.; Schade, T.G. Random Demands, Travel Times and Water Quality in Dead-Ends, Prepared for American Water Works Association Research Foundation; Report No. 294; American Water Works Association Research Foundation: Denver, CO, USA, 2003.

(C) 2017 by the author. Licensee MDPI, Basel, Switzerland. This article is an open access article distributed under the terms and conditions of the Creative Commons Attribution (CC BY) license (http://creativecommons.org/licenses/by/4.0/). 